BI-TP 2014/18

\title{
Hadron Freeze-Out and Unruh Radiation
}

\author{
Paolo Castorina ${ }^{\mathrm{a}, \mathrm{b}}$, , Alfredo Iorio ${ }^{\mathrm{c}}$ and Helmut Satz \\ a: Dipartimento di Fisica ed Astronomia, Universita' di Catania, Italy \\ b: INFN sezione di Catania, Catania, Italy \\ c: Faculty of Mathematics and Pysics, Charles Univeristy in Prague, Czech Republic \\ d: Fakultät für Physik, Universität Bielefeld, Germany
}

\begin{abstract}
We consider hadron production in high energy collisions as an Unruh radiation phenomenon. This mechanism describes the production pattern of newly formed hadrons and is directly applicable at vanishing baryonchemical potential, $\mu \simeq 0$. It had already been found to correctly yield the hadronisation temperature, $T_{h}=\sqrt{\sigma / 2 \pi} \simeq 165 \mathrm{MeV}$ in terms of the string tension $\sigma$. Here we show that the Unruh mechanism also predicts hadronic freeze-out conditions, giving $s / T_{h}^{3}=3 \pi^{2} / 4 \simeq 7.4$ in terms of the entropy density $s$ and $\langle E\rangle /\langle N\rangle=\sqrt{2 \pi \sigma} \simeq 1.09$ for the average energy per hadron. These predictions provide a theoretical basis for previous phenomenological results and are also in accord with recent lattice studies.
\end{abstract}


The relative abundances of hadrons produced in $e^{+} e^{-}$annihilation [1 3], in hadron-hadron interactions [3,4] and in the collisions of heavy nuclei [5-11], are well described by an ideal hadronic resonance gas at fixed temperature $T$ and baryonchemical potential $\mu$. In the high energy, low baryon density regime, from around $20 \mathrm{GeV}$ up to the $\mathrm{TeV}$ range, such a description leads to a universal chemical freeze-out temperature $T_{H} \simeq 165 \pm 5 \mathrm{MeV}[12]$. This temperature moreover agrees well with lattice QCD results at low or vanishing $\mu$ [13]. Extensions to larger baryon density have led to various phenomenological proposals for freeze-out conditions [14-20]. While these models indeed describe the present data quite well, neither the crucial parameters nor the conceptual forms appear to have a basic theoretical justification. The aim of this paper is to show that for low or vanishing $\mu$, such a basis indeed exists.

The universality of the observed hadronisation temperature suggests a common origin for all high energy collisions, and it was recently proposed [21] that high energy thermal hadron production is the QCD counterpart of Unruh radiation [22], emitted at the event horizon due to color confinement. Let us briefly recall the relevant formalism.

The basic mechanism of Unruh radiation is tunnelling through the confining event horizon. It is most simply illustrated by hadron production through $e^{+} e^{-}$annihilation into a $q \bar{q}$ pair; see Fig. 1 .
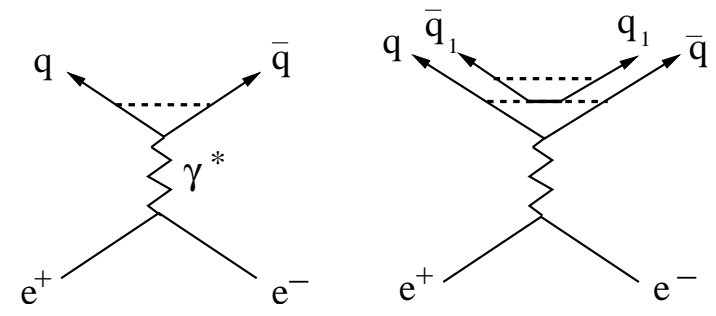

Figure 1: Quark formation in $e^{+} e^{-}$annihilation

The attempt to separate ends for the initial $q \bar{q}$ pair at a distance $R$, when each quark hits the confinement horizon, i.e., when it reaches the end of the binding string; the separation can now continue only if a further quark-antiquark system is excited from the vacuum. Although the new pair $q_{1} \bar{q}_{1}$ is at rest in the overall center of mass system, each of its constituents has a transverse momentum $k_{T}$, determined by the uncertainty relation in terms of the transverse dimension of the string flux tube. String theory [23] gives for the basic thickness

$$
r_{T}=\sqrt{2 / \pi \sigma}
$$

leading to

$$
k_{T}=\sqrt{\pi \sigma / 2}
$$

The maximum separation distance $R$ is thus specified by

$$
\sigma R=2 \sqrt{m_{q}^{2}+k_{T}^{2}}=2 k_{T},
$$

where we have taken $m_{q}=0$ for the quark mass. From this we obtain

$$
R=\sqrt{2 \pi / \sigma}
$$


as the string breaking distance. The departing quark $q$ now pulls the newly formed $\bar{q}_{1}$ along, giving it an acceleration [21,24]

$$
a=\sqrt{2 \pi \sigma} .
$$

The $q_{1} \bar{q}_{1}$ pair eventually suffers the same fate as the $q \bar{q}$ pair: it is separated up to its confinement horizon, where it again excites a new pair, which is now emitted as Unruh radiation of temperature

$$
T_{h}=a / 2 \pi=\sqrt{\sigma / 2 \pi}
$$

This process is sequentially repeated until the energy of the initial "driving" quarks $q$ and $\bar{q}$ is exhausted.

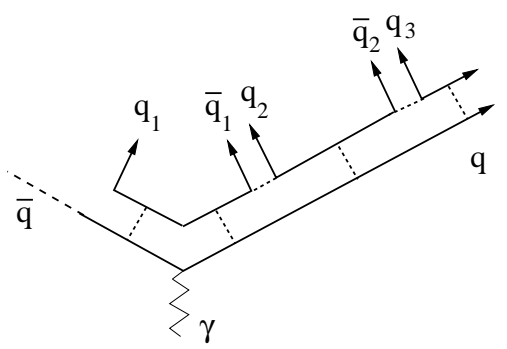

Figure 2: Sequential quark formation in $e^{+} e^{-}$annihilation

The case of $e^{+} e^{-}$annihilation corresponds to baryochemical potential, $\mu=0$. Here one finds the average value $\sigma \simeq 0.19 \pm 0.03 \mathrm{GeV}^{2}$ [25]29], which with eq. (6) then leads to

$$
T_{h}(\mu=0)=\sqrt{\sigma / 2 \pi} \simeq 175 \pm 15 \mathrm{MeV}
$$

for the freeze-out temperature at $\mu=0$, in good agreement with the results of both the mentioned resonance gas analyses [14-16] and lattice QCD studies [13]. The fundamental mechanism in the Unruh scenario is quark acceleration leading to string breaking with the resuling pair production, as specified by eq. (33). As long as we assume a vanishing quark mass, the only dimensional parameter in the entire formalism then is the string tension $\sigma$.

The Unruh mechanism provides a theoretical basis for the production of newly formed hadrons in high energy collisions. It does not address the role of the nucleons already present in the initial state of heavy ion collisions, and as such it can a priori describe the freeze-out process only as long as there are no significant baryon-density effects, i.e., only for $\mu \simeq 0_{1}^{1}$ Here, however, it makes more well-defined predicitions than just eq. (7).

The energy of the pair produced by string breaking, i.e., of the newly formed hadron, is from eq's. (2,3) given by

$$
E_{h}=\sigma R=\sqrt{2 \pi \sigma}
$$

In the central rapidity region of high energy collisions, one has $\mu \simeq 0$, so that $E_{h}$ is in fact the average energy $\langle E\rangle$ per hadron, with an average number $\langle N\rangle$ of newly produced hadrons. Hence we obtain

$$
\frac{\langle E\rangle}{\langle N\rangle}=\sqrt{2 \pi \sigma} \simeq 1.09 \pm 0.08,
$$

\footnotetext{
${ }^{1}$ An attempt to address finite $\mu$ effects is given in [30].
} 
in accord with the phenomenological fit obtained from the species abundances in high energy collisions [14 16].

Next, we turn to the entropy. Because of the event horizon caused by color confinement, this is necessarily an entanglement entropy, caused by quantum field modes on both sides of the horizon. Its general form is [31, 32]

$$
S_{\mathrm{ent}}=\alpha \frac{A}{r^{2}},
$$

where $A$ is the area of the event horizon, $r$ the scale of characteristic quantum fluctuations and $\alpha$ an undetermined numerical constant. This expression shares the holographic structure 2 with the Bekenstein-Hawking entropy [35, 36] for a black hole,

$$
S_{\mathrm{BH}}=\frac{1}{4} \frac{A_{\mathrm{BH}}}{r_{P}^{2}}
$$

where $A_{\mathrm{BH}}=4 \pi R_{S}^{2}$ denotes the surface area of the hole and $R_{S}=2 G M / c^{2}$ is its Schwarzschild radius. The quantity $r_{P}=\sqrt{\hbar G / c^{3}}$ is the Planck length, setting the smallest possible fluctuation scale.

The Bekenstein-Hawking relation (11) also holds in the case of an accelerated observer (Rindler spacetime), where it corresponds to the near-horizon approximation of a black hole spacetime [37]. Here we take it to be valid also in our case, where gravity is not involved and the entire entropy is of the entanglement type. The scale of the characteristic quantum fluctuations is now given by eq. (1), and we obtain

$$
S_{h}=\frac{1}{4} \frac{A_{h}}{r_{T}^{2}}=\frac{1}{4} \frac{4 \pi R^{2}}{r_{T}^{2}}
$$

for the entropy in hadron production. The parameter $R$ is given by eq. (4), and the smallest fluctuation scale is the transverse string thickness (1). Inserting these expressions into eq. (12) gives for the entropy associated to hadron production

$$
S_{h}=\pi^{3}
$$

i.e., it becomes a pure number, and also the entropy density divided by $T^{3}$ remains dimensionles, giving

$$
\frac{s}{T^{3}}=\frac{S_{h}}{(4 \pi / 3) R^{3} T^{3}}=\frac{3 \pi^{2}}{4} \simeq 7.4
$$

as freeze-out condition in terms of $s(T)$ and $T$. This result is in accord with the value obtained for $s / T^{3}$ from species abundance analyses in terms of the ideal resonance gas model [19, 20]. In Fig. 3] we see that it agrees as well with the most recent lattice QCD studies [13].

We close with two remarks. First, we comment on freeze-out for larger baryochemical potential $\mu$. This implies the presence of nucleons in the initial state, not formed by the collision, and their interaction with the newly formed ones. Such dynamical effects are not

\footnotetext{
${ }^{2}$ Holography of entanglement entropy is a quite general result, see [33, 34].
} 


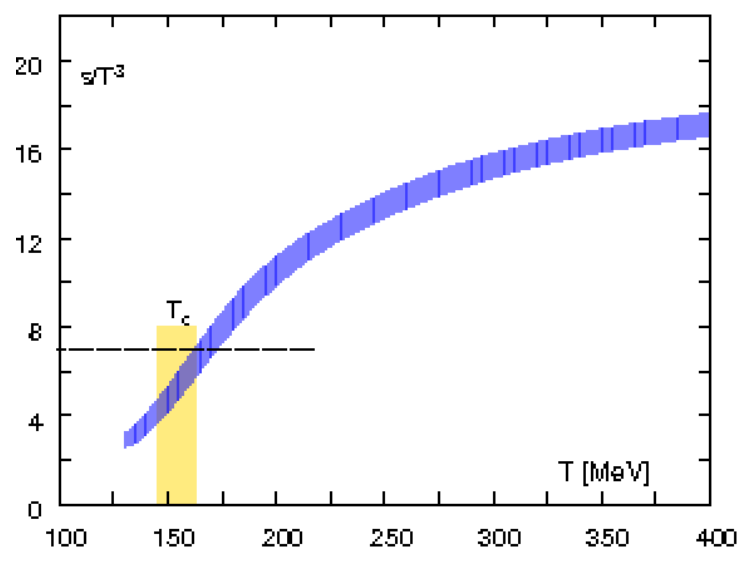

Figure 3: Lattice results [13] for $s / T^{3}$ as function of the temperature

included in the Unruh formalism and hence cannot be addressed here. We therefore cannot answer the tantalizing question of why the conditions $\langle E /\rangle N \simeq 1.08$ or $s / T^{3}=7.4$, valid for $\mu \simeq 0$, appear to remain as valid also for increasing $\mu$. This must imply an intricate interplay the decreasing role of secondary mesons vs. the increasing role of the nucleons present already in the initial state.

Secondly, we want to emphasize that besides the obvious importance of addressing issues of QCD phenomenology, the Unruh phenomenon appearing here is of great importance in its own right. Unruh phenomena have eluded direct observation since decades, and having them here at our disposal in the laboratory may well help in solving some of the long-standing open problems of theoretical aspects in fundamental physics, see, e.g., [38. One such issue might be the clarification of the entanglement nature of the BekensteinHawking entropy.

\section{Acknowledgements}

We thank S. Mukherjee, BNL, for providing us with the lattice results of the HotQCD Collaboration (Fig. 3). P. C. thanks the Faculty of Physics, Bielefeld University, for the kind hospitality. A. I. wishes to thank the Department of Physics and Astronomy of Catania University, and INFN Sezione di Catania, for the kind hospitality, and acknowledges the Czech Science Foundation (GAČR), Contract No. 14-07983S, for support. H. S. very belatedly thanks A. Tawfik for asking him many years ago why hadron production data lead to $s / T^{3}=7$.

\section{References}

[1] F. Becattini, Z. Phys. C69 (1996) 485.

[2] F. Becattini, Universality of thermal hadron production in $p p, p \bar{p}$ and $e^{+} e^{-}$collisions, in Universality features in multihadron production and the leading effect, Erice 1966, World Scientific, Singapore (1998) 74-104; arXiv:hep-ph/9701275.

[3] F. Becattini and G. Passaleva, Eur. Phys. J. C23 (2002) 551. 
[4] F. Becattini and U. Heinz, Z. Phys. C76 (1997) 268.

[5] J. Cleymans et al., Phys. Lett. B 242 (1990) 111.

[6] J. Cleymans and H. Satz, Z. Phys. C57 (1993) 135.

[7] K. Redlich et al., Nucl. Phys. A 566 (1994) 391.

[8] P. Braun-Munzinger et al., Phys. Lett. B344 (1995) 43.

[9] F. Becattini, M. Gazdzicki and J. Sollfrank, Eur. Phys. J. C5 (1998) 143.

[10] F. Becattini et al., Phys. Rev. C64 (2001) 024901.

[11] P. Braun-Munzinger, K. Redlich and J. Stachel, in Quark-Gluon Plasma 3, R. C. Hwa and X.-N Wang (Eds.), World Scientific, Singapore 2003.

[12] F. Becattini, Nucl. Phys. A702 (2001) 336c.

[13] See A. Bazazov et al. (HotQCD Collaboration), arXiv:1407.6387, and further references given there.

[14] J. Cleymans and K. Redlich, Phys. Rev. Lett. 81 (1998) 5284.

[15] J. Cleymans and K. Redlich, Phys. Rev. C 61 (1999) 054908.

[16] J. Cleymans et al., arXiv:hep-ph/0511094

[17] P. Braun-Munzinger and J. Stachel, J. Phys. G 28 (2002) 1971.

[18] V. Magas and H. Satz, Eur. Phys. J. C32 (2003) 115.

[19] J. Cleymans et al., Phys. Lett. B 615 (2005) 50.

[20] A. Tawfik, J. Phys. G 31 (2005) S1105; hep-ph/0507252 and hep-ph/050824.

[21] P. Castorina, D. Kharzeev and H. Satz, Eur. Phys. J. C52 (2007) 187.

[22] W. G. Unruh, Phys. Rev. D 14 (1976) 870.

[23] M. Lüscher, G. Münster and P. Weisz, Nucl. Phys. B 180 (1981) 1.

[24] F. Becattini et al., Eur. Phys. J. C56 (2008) 493.

[25] F. J. Yndurain, Theory of Quark and Gluon Interactions, Springer Verlag Berlin, 1999.

[26] N. Brambilla et al., CERN Yellow Report CERN-2005-005

[27] C. Aubin et al. (MILC Collaboration), Phys. Rev. D70 (2004) 094505.

[28] A. Gray et al., Phys. Rev. D72 (2005) 0894507.

[29] M. Cheng et al., arXiv:hep-lat/0608013 
[30] P. Castorina, D. Grumiller and A. Iorio, Phys. Rev. D 77 (2008) 124034.

[31] H. Terashima, Phys. Rev. D61 (2000) 104016.

[32] A. Iorio, G. Lambiase, G. Vitiello, Ann. Phys. 309 (2004) 151.

[33] M. Srenidcky, Phys.Rev.Lett. 71 (1993) 666.

[34] S. N. Solodukhin, Liv. Rev. Rel. 14 (2011) 8.

[35] J. D. Bekenstein, Phys. Rev. D23 (1973) 2333.

[36] S. Hawking, Nature 248 (1974) 30.

[37] R. Laflamme, Phys. Lett. B 196 (1987) 449.

[38] A. Iorio, Eur. Phys. J. Plus 127 (2012) 156. 